\title{
THE COMPACTNESS OF THE RIEMANN MANIFOLD OF AN ABSTRACT FIELD OF ALGEBRAIC FUNCTIONS
}

\section{OSCAR ZARISKI}

1. The existence of finite resolving systems. In an earlier paper ${ }^{1}$ we have announced the result that the existence of a resolving system of the Riemann manifold of an abstract field of algebraic functions (in any number of variables) or-what is the same-the local uniformization theorem ${ }^{2}$ implies the existence of finite resolving systems of the Riemann manifold. We have proved this result for algebraic surfaces by arithmetic considerations. ${ }^{1}$ The proof for the general case of varieties, which at that time was in our possession, ${ }^{3}$ and which we have promised to publish in a subsequent paper, was of similar nature, that is, it was based upon considerations involving the structure of certain infinite sequences of quotient rings. However, we have succeeded lately in finding a much simpler proof which is based on topological considerations.

Let $\Sigma$ be a field of algebraic functions of several variables, over an arbitrary ground field $k$. By the Riemann manifold $M$ of $\Sigma$ we mean the totality of places of $\Sigma$, that is, the totality of zero-dimensional valuations $v$ of $\Sigma / k$. If $V$ is a projective model of $\Sigma / k$, and if $H$ is any subset of $V$, we denote by $N(H)$ the subset of $\boldsymbol{M}$ consisting of those valuations $\mathfrak{b}$ which have center in $H$. By a resolving system of $\boldsymbol{M}$ we mean a collection $\mathfrak{B}=\left\{V_{a}\right\}$ of projective models (finite or infinite in number) with the property that for any $v$ in $M$ there exists a $V_{a}$ in $\mathfrak{B}$ such that the center of $v$ on $V_{a}$ is a simple point.

The topology which we introduce in $M$ is simply this: we choose as a basis for the closed sets of $M$ the sets $N(W)$, where $W$ is any algebraic subvariety of any projective model of $\Sigma$. We prove that if topologized in this fashion, the set $M$ is a compact topological space. From this the result announced above follows immediately. For if $\left\{V_{a}\right\}$ is a resolving system, and if we denote by $S_{a}$ the singular locus of $V_{a}$, then $N\left(V_{a}-S_{a}\right)$ is an open set and $\left\{N\left(V_{a}-S_{a}\right)\right\}$ is an open covering of $\boldsymbol{M}$.

Received by the editors April 10, 1944.

${ }^{1}$ A simplified proof for the resolution of singularities of an algebraic surface, Ann. of Math. vol. 43 (1942) p. 583.

2 See loc. cit. footnote 1.

${ }^{3}$ That proof was presented by us at a seminar in algebraic geometry at Johns Hopkins in 1942.

-We use the term compact in the same sense as it is used by S. Lefschetz in his Algebraic topology (Amer. Math. Soc. Colloquium Publications, vol. 27, 1942). The old term is bicompact. 
Hence this covering contains a finite subcovering $\left\{N\left(V_{i}-S_{i}\right)\right\}$, $i=1,2, \cdots, m$, and this means that $\left\{V_{1}, V_{2}, \cdots, V_{m}\right\}$ is a finite resolving system of $\boldsymbol{M}$.

The proof of compactness of $M$ given in the next section is based in part on some simple algebro-geometric considerations, and in part on a theorem of Steenrod ${ }^{5}$ on the compactness of the limit space of an inverse system of compact $T_{1}$-spaces.

2. The Riemann manifold as the limit space of an inverse system. Let $\mathfrak{B}=\left\{V_{a}\right\}$ be the collection of all projective models of $\Sigma / k$. By a point of $V_{a}$ we mean a zero-dimensional prime ideal in a suitable coördinate ring of $V_{a}$, or, in other terms, a point is a prime onedimensional homogeneous ideal in the ring of homogeneous coördinates of the general point of $V_{a}$. This defines $V_{a}$ set-theoretically as a set of points. We topologize $V_{a}$ by choosing as closed sets the algebraic subarieties of $V_{a}$. It is obvious that $V_{a}$ then becomes a compact topological space in which points are closed sets (whence $V_{a}$ is a $T_{1}$-space; however, it is not a Hausdorff space).

If $V_{a}$ and $V_{b}$ are two projective models of $\Sigma / k$, we denote by $\pi_{a}^{b}$ the transformation of $V_{b}$ onto $V_{a}$ defined by the birational correspondence between $V_{a}$ and $V_{b}$. We define a partial ordering < of the collection $\mathfrak{B}$ as follows: $V_{a} \prec V_{b}$ if whenever $P_{a}$ and $P_{b}$ are corresponding points of $V_{a}$ and $V_{b}$ under $\pi_{a}^{b}$ then $Q\left(P_{a}\right) \subseteq Q\left(P_{b}\right)$. Here $Q(P)$ denotes the quotient ring of $P$. It is clear that if $V_{a}<V_{b}$ then $\pi_{a}^{b}$ is a single-valued continuous and closed mapping. Moreover, if $V_{a}$ and $V_{b}$ are arbitrary projective models of $\Sigma / k$, and if $V_{c}$ denotes the join ${ }^{6}$ of $V_{a}$ and $V_{b}$, then $V_{a} \prec V_{c}$ and $V_{b} \prec V_{c}$. Hence we have here an inverse system $\left\{V_{a} ; \pi_{a}^{b} \mid V_{a} \prec V_{b}\right\}$ of compact $T_{1}$-spaces. Let $M$ be the limit space of the system. By Steenrod's theorem $\boldsymbol{M}$ is compact. Every point $P^{*}$ of $M$ represents an infinite collection of points $\left\{P_{a}\right\}$, $P_{a} \in V_{a}, V_{a} \in \mathfrak{B}$, with the property that if $V_{a}<V_{b}$ then $Q\left(P_{a}\right) \subseteq Q\left(P_{b}\right)$. We shall denote by $\pi_{a}^{*}$ the mapping $P^{*} \rightarrow P_{a}$ of $M$ into $V_{a}$. If $V_{a}$ is any projective model of $\Sigma / k$ and if $W$ is any algebraic subvariety of $V_{a}$, then $\left(\pi_{a}^{*}\right)^{-1} W$ is a closed subset of $\boldsymbol{M}$, and the closed sets obtained in this fashion form a basis for the closed subsets of $M$.

The compactness of the Riemann manifold of $\Sigma / k$ and the implications stated in the preceding section are immediate consequences of the following theorem.

TheOREM. There is $a(1,1)$ correspondence between the points $P^{*}$

\footnotetext{
${ }^{5}$ N. E. Steenrod, Universal homology groups, Amer. J. Math. vol. 58 (1936) p. 666.

- See our paper Foundations of a general theory of birational correspondences, Trans. Amer. Math. Soc. vol. 53 (1943) p. 516.
} 
of $M$ and the zero-dimensional valuations $v$ of the field $\Sigma / k$. If $P^{*}$ and $v$ are corresponding elements, and if $V_{a}$ is any projective model of $\Sigma / k$, then $\pi_{a}^{*} P^{*}$ is the center of $v$ on $V_{a}$.

Proof. Let $v$ be a zero-dimensional valuation of $\Sigma / k$ and let $P_{a, v}$ be the center of $v$ on any given projective model $V_{a}$ of $\Sigma / k$. For any two projective models $V_{a}, V_{b}$ it is then true that $P_{a, v}$ and $P_{b, v}$ are corresponding points in the birational correspondence $\pi_{a}^{b}$. Hence $P_{v}^{*}=\left\{P_{a, v}\right\}$ is a point of $M$. Thus every zero-dimensional valuation $v$ determines uniquely a point $P_{v}^{*}$ of $M$.

If $v_{1}$ and $v_{2}$ are two distinct zero-dimensional valuations, then there exists at least one projective model $V_{a}$ such that $P_{a, v_{1}} \neq P_{a_{v} v_{2}}$. Hence if $v_{1} \neq v_{2}$ than $P_{v_{1}}^{*} \neq P_{v_{2}}^{*}$.

Now let $P^{*}$ be an arbitrary point of $M, P^{*}=\left\{P_{a}\right\}$. We denote by $\mathfrak{B}$ the least ring containing the quotient rings $Q\left(P_{a}\right)$. Let $V_{b}$ be a fixed projective model of $\Sigma / k$ and let $P_{b}=\pi_{b}^{*} P^{*}$. We assert that if $\omega$ is $a$ non-unit in $Q\left(P_{b}\right)$ then $1 / \omega \in \mathfrak{B}$. For assume that $1 / \omega \in \mathfrak{B}$. Then $1 / \omega$ will belong to the ring generated by a finite number of quotient rings $Q\left(P_{a}\right)$, say $Q\left(P_{a_{1}}\right), Q\left(P_{a_{2}}\right), \cdots, Q\left(P_{a_{m}}\right)$. Let $V_{c}$ be the join of the varieties $V_{b}, V_{a_{1}}, V_{a_{2}}, \cdots, V_{a_{m}}$ and let $P_{c}=\pi_{c}^{*} P^{*}$. Since $\pi_{a i}{ }^{*} P^{*}=P_{a i}$ and $\pi_{b}^{*} P^{*}=P_{b}$, we have $\pi_{a_{i}}^{c} P_{c}=P_{a i}$ and $\pi_{b}^{c} P_{c}=P_{b}$, and hence $Q\left(P_{a i}\right) \subseteq Q\left(P_{c}\right), Q\left(P_{b}\right) \subseteq Q\left(P_{c}\right)$. Therefore $1 / \omega \in Q\left(P_{c}\right)$. This is a contradiction since any non-unit of $Q\left(P_{b}\right)$ is obviously also a non-unit in $Q\left(P_{c}\right)$.

We have therefore shown that $\mathfrak{B}$ is a proper ring (not a field). We now show that $\mathfrak{B}$ is a valuation ring. For this it is sufficient to show that if $\xi$ is any element of $\Sigma$ then either $\xi \in \mathfrak{B}$ or $1 / \xi \in \mathfrak{B}$. We consider again a fixed projective model $V_{b}$ of $\Sigma / k$. We select a system of nonhomogeneous coördinates $x_{1}, x_{2}, \cdots, x_{n}$ of the general point of $V_{b}$ in such a fashion that the point $P_{b}\left(=\pi_{b}^{*} P^{*}\right)$ is at finite distance with respect to these coördinates. Let $V_{d}$ be the projective model whose general point has as nonhomogeneous coördinates the elements $x_{1}, x_{2}, \cdots, x_{n}, \xi$. If the point $P_{d}\left(=\pi_{d}^{*} P^{*}\right)$ is at finite distance with respect to these coördinates, then $\xi \in Q\left(P_{d}\right) \subseteq \mathfrak{B}$. If $P_{d}$ is a point at infinity, we observe first of all that in the above proof of our assertion $1 / \omega \notin \mathbb{B}$ we have shown incidentally the following: if $V_{a}$ and $V_{b}$ are any two projective models of $\Sigma / k$ and if $\pi_{a}{ }^{*} P^{*}=P_{a}$ and $\pi_{b}^{*} P^{*}=P_{b}$, then $P_{a}$ and $P_{b}$ are corresponding points of the birational correspondence between $V_{a}$ and $V_{b}$. For on the join $V_{c}$ of $V_{a}$ and $V_{b}$ we have the point $P_{c}=\pi_{c}^{*} P^{*}$ and the relations $Q\left(P_{c}\right) \supseteq Q\left(P_{a}\right)$, $Q\left(P_{c}\right) \supseteq Q\left(P_{b}\right)$. These relations show that if $v$ is any zero-dimensional valuation whose center on $V_{c}$ is the point $P_{c}$, then the center of $v$ on $V_{a}$ is $P_{a}$ and its center on $V_{b}$ is $P_{b}$. Hence $P_{a}$ and $P_{b}$ are indeed 
corresponding points. ${ }^{7}$ With this observation in mind, let $v$ be a zerodimensional valuation whose center on $V_{b}$ is the point $P_{b}$ and whose center on $V_{d}$ is the point $P_{d}$. Since $P_{b}$ is at finite distance, we have $v\left(x_{i}\right) \geqq 0, i=1,2, \cdots, n$. Since $P_{d}$ is at infinity, we must have $v(\xi)<0$. Hence $v(1 / \xi)>0, v\left(x_{i} / \xi\right)>0$, and this shows that if we take $1 / \xi, x_{1} / \xi, \cdots, x_{n} / \xi$ as nonhomogeneous coördinates of the general point of $V_{d}$, then $P_{d}$ is at finite distance. Hence $1 / \xi \in Q\left(P_{d}\right) \subseteq \mathscr{B}$. This completes the proof of our assertion that $\mathfrak{B}$ is a valuation ring.

Let $v$ be the valuation defined by the valuation ring $\mathfrak{B}$. We assert that $v$ is zero-dimensional. For let $v$ be of dimension $s$. We can find a projective model $V_{b}$ on which the center of $v$ is an $s$-dimensional variety $W$. If $P_{b}=\pi_{b}^{*} P^{*}$, then $Q\left(P_{b}\right) \subseteq \mathscr{B}$ and this implies that $P_{b} \in W .{ }^{8}$ If $s>0$, then we can find a non-unit $\omega$ in $Q\left(P_{b}\right)$ such that $\omega \neq 0$ on $W$, whence $1 / \omega \in Q(W) \subseteq \mathfrak{B}$, a contradiction. Hence $s=0$, as asserted.

The above relation $P_{b} \in W$ implies now $P_{b}=W$. This is true for any projective model $V_{b}$, that is, the center of $v$ on any projective model $V_{b}$ is the point $P_{b}=\pi_{b}^{*} P^{*}$. This completes the proof of the theorem.

3. A generalization. Infinite direct products of projective lines. The idea of topologizing an algebraic variety $V$ by choosing as closed sets the algebraic subvarieties of $V$ can be used with good effect in order to topologize the set $M^{*}$ of all homomorphic mappings of any abstract field $A$ into another abstract field $K$. In this general case we are dealing essentially with a generalization of the concept of the Riemann manifold of a field of algebraic functions (see the Remark at the end of the paper). We begin with some topological preliminaries.

Let $\left\{R_{a}\right\}$ be a system of compact topological spaces indexed by a set $A=\{a\}$. We assume that each $R_{a}$ is a $T_{1}$-space; that is, that the points of $R_{a}$ are closed sets. Elements of $A$ shall be denoted by small Latin letters, $a, b, c, \cdots$; subsets of $A$ shall be denoted by small Greek letters, $\alpha, \beta, \gamma, \cdots$. If $\alpha$ is a subset of $A$ we shall denote by $R_{\alpha}$ the direct product $P_{a \in \alpha} R_{a}$. If $\alpha \subset \beta$ we denote by $\pi_{\alpha}^{\beta}$ the projection of $R_{\beta}$ onto $R_{\alpha}$. Finally, elements of $R_{a}$ and $R_{\alpha}$ shall be denoted by $x_{a}, y_{a}, z_{a}, \ldots$ and by $x_{\alpha}, y_{\alpha}, z_{\alpha}, \ldots$ respectively. If $a \in \alpha$ and if $\pi_{a}^{\alpha} x_{\alpha}=x_{a}$, then $x_{\alpha}$ shall be referred to as the $a$-component of $x_{\alpha}$.

We assume that for each finite subset $\alpha$ of $A$ a topology has been assigned to $R_{\alpha}$ and that the following three conditions are satisfied: (1) $R_{\alpha}$ is a compact topological space; (2) if $\alpha \subset \beta$ then $\pi_{\alpha}^{\beta}$ is a closed

${ }^{7}$ See our definition of corresponding points of a birational transformation, loc. cit. footnote 6, p. 505.

8 See loc. cit. footnote 6, Theorem 3, p. 497. 
mapping (mapping = single-valued continuous transformation); (3) if $\alpha$ is a set with one element a then the topology assigned to $R_{\alpha}$ is exactly the topology of $R_{a}$. It is clear that in virtue of these two conditions $R_{\alpha}$ is a $T_{1}$-space. For if $x_{a}$ is the $a$-component of $x_{\alpha}$, then $\left(\pi_{a}^{\alpha}\right)^{-1} x_{a}$ is closed and $x_{\alpha}$ is the intersection of the closed sets $\left(\pi_{a}^{\alpha}\right)^{-1} x_{a}, a \in \alpha$.

If we consider only finite subsets $\alpha$ of $A$ and if we define a partial ordering in the collection $\left\{R_{\alpha}\right\}$ by setting $R_{\alpha}<R_{\beta}$ if $\alpha \subset \beta$, then we have an inverse system $\left\{R_{\alpha} ; \pi_{\alpha}^{\beta}\right\}$. It is clear that set-theoretically the limit space $R^{*}$ of the system coincides with the direct product $R^{*}=P_{a \in A} R_{a}$. However, the topology in $R^{*}$ is not necessarily the usual topology of the product space, for our topology in $R^{*}$ depends not only on the topology of each factor $R_{a}$ but also on the topology which has been assigned to each $R_{\alpha}$, for $\alpha$ any finite subset of $A$.

Our space $R^{*}$ is compact, by Steenrod's theorem. We are dealing here with a special case of Steenrod's theorem, and the proof of the compactness of $R^{*}$ can be somewhat simplified. For this reason, and also for the convenience of the reader, we shall include here a proof of the compactness of $R^{*}$.

We have to show that if a family of closed sets in $R^{*}$ has the finite intersection property (that is, if every finite subfamily has a nonempty intersection), then the intersection of the entire family is non-empty. It will be sufficient to prove this for families of basic closed sets $F_{\alpha}^{*}, F_{\alpha}^{*}=\pi_{\alpha}^{-1} F_{\alpha}$, where $F_{\alpha}$ denotes a closed set in $R_{\alpha}$ and where $\pi_{\alpha}$ is the projection of $R^{*}$ onto $R_{\alpha}$. Let then $\left\{F_{\alpha}^{*}\right\}$ be a family $\mathfrak{F}$ of basic closed sets which has the finite intersection property. By Zorn's lemma the family $\left\{F_{\alpha}^{*}\right\}$ is contained in a maximal family $\left\{G_{\alpha}{ }^{*}\right\}$ of basic closed sets which has the finite intersection property. It will be sufficient to show that $\cap G_{\alpha}{ }^{*}$ is non-empty. We shall therefore assume that our original family $\left\{F_{\alpha}^{*}\right\}$ is not contained properly in another family of basic closed sets which has the finite intersection property. ${ }^{\circ}$

We first observe that the intersection of any finite collection of basic closed sets is itself a basic closed set. For let $\left\{\pi_{\alpha i}^{-1} F_{\alpha i}\right\}$ be a finite collection of basic closed sets. We put $\alpha=\bigcup \alpha_{i}, F_{\alpha}=\bigcap\left(\pi_{\alpha_{i}}^{\alpha}\right)^{-1} F_{\alpha i}$. Then it is clear that $\bigcap \pi_{\alpha i}^{-1} F_{\alpha i}=\pi_{\alpha}^{-1} F_{\alpha}$.

In virtue of this remark and in virtue of the maximality property

-The idea of passing to a maximal family is taken from the proof of Tychonoff's theorem as given in Lefschetz, Algebraic topology, p. 19. There is only this difference: the maximal family in Lefschetz is not a family of closed sets, while ours is. This modification of the proof succeeds because we restrict ourselves to families of basic closed sets and because in our case the mappings $\pi_{\alpha}^{\beta}$ are closed. 
of the given family $\mathfrak{F}$, it follows that every finite intersection of sets in $\mathfrak{F}$ is again in the family $\mathfrak{F}$.

For any element $a$ in $A$ and for any member $F_{\alpha}{ }^{*}$ in $\mathfrak{F}$ let $\pi_{a} F_{\alpha}{ }^{*}=F_{\alpha, a}$. If $a \notin \alpha$ then it is clear that $F_{\alpha, a}=R_{a}$, for then the $a$-component of the points of $F_{\alpha}{ }^{*}$ is not restricted. If $a \in \alpha$ and if $F_{\alpha}{ }^{*}=\pi_{\alpha}^{-1} F_{\alpha}$, then $F_{\alpha, a}=\pi_{a}^{\alpha} F_{\alpha}$. In either case $F_{\alpha, a}$ is a closed set in $R_{a}$, for we have assumed that $\pi_{\alpha}^{\beta}$ is closed whenever $\alpha \subset \beta$. For a given $a$ the family $\mathfrak{F}_{a}$ of closed sets $\left\{F_{\alpha, a}\right\}$ has the finite intersection property. Since $R_{a}$ is compact, the intersection $\bigcap_{\alpha} F_{\alpha, a}$ is non-empty. Let $x_{a}$ be a point common to all the sets in $\mathfrak{F}_{a}$. Then $\pi_{a}^{-1} x_{a}$ is a basic closed set (since $R_{a}$ is a $T_{1}$-space) which meets every set $F_{\alpha}^{*}$ in $\mathfrak{F}$. Consequently $\pi_{a}^{-1} x_{a} \in \mathfrak{F}$, $x_{a} \in F_{a}$, and the intersection $\bigcap_{\alpha} F_{\alpha, a}$ consists only of the point $x_{a}$.

Let then $x=\left\{x_{a}\right\}$, where $x_{a}=\bigcap_{\alpha} F_{\alpha, a}$. We show that $x$ is a common point of the sets $F_{\alpha}{ }^{*}$ in $\mathfrak{F}$. Since $\pi_{a}^{-1} x_{a} \in \mathfrak{F}$, for any $a$, it follows that $\bigcap_{a \in \alpha_{\alpha}} \pi_{a}^{-1} x_{\alpha} \in \mathfrak{F}$, that is, $\pi_{\alpha}^{-1} x_{\alpha} \in \mathfrak{F}$, where $x_{\alpha}=\pi_{\alpha} x$. Therefore $\pi_{\alpha}^{-1} x_{\alpha}$ meets $F_{\alpha}^{*}$, that is, $\pi_{\alpha}^{-1} F_{\alpha}$; hence $x_{\alpha} \in F_{\alpha}$ and $x \in \pi_{\alpha}^{-1} x_{\alpha} \subset \pi_{\alpha}^{-1} F_{\alpha}=F_{\alpha}^{*}$, q.e.d.

Now let $K$ be a fixed abstract field and let the sets $R_{a}$ be projective lines over $K$, so that the points of each set $R_{a}$ are in $(1,1)$ correspondence with the elements of $K$ together with the symbol $\infty$. We topologize $R_{a}$ by choosing as closed sets the finite subsets of $R_{a}$. Then each $R_{a}$ becomes a compact topological $T_{1}$-space.

We still have to topologize each set $R_{\alpha}$, for $\alpha$ a finite subset of $A$. For this purpose we introduce on each line $R_{a}$ a pair of homogeneous coördinates $x_{a 1}, x_{a 2}$ and we define an algebraic variety $V_{\alpha}$ by the following parametric equations (in which the $X_{(e)}^{(\alpha)}$ denote the homogeneous coördinates of the general point of $V_{\alpha}$ ):

$$
\rho \cdot X_{\epsilon_{1} \epsilon_{2}}^{(\alpha)} \cdots \epsilon_{n}=x_{a_{1} \epsilon_{1}} x_{a_{2} \epsilon_{2}} \cdots x_{a_{n} \varepsilon_{n}},
$$

where $\alpha=\left\{a_{1}, a_{2}, \cdots, a_{n}\right\}$ and where each $\epsilon_{j}$ can take the values 1 or 2. It is well known that $V_{\alpha}$ is a Segre variety, of dimension $n$, immersed in a projective space of dimension $2^{n}-1$. The points of $V_{\alpha}$ are in $(1,1)$ correspondence with $n$-tuples of ratios $\left\{x_{a 2} / x_{a 1}\right\}, a \in \alpha$, that is, with the points of the direct product $R_{\alpha}=R_{a_{1}} \times R_{a_{2}} \times \cdots$ $\times R_{a_{n}}$. It should be noted that here we only consider points $X^{\alpha}$ whose homogeneous coördinates are in $K$. We topologize $V_{\alpha}$ by choosing as closed sets the algebraic subvarieties of $V_{\alpha}$. Then it is clear that each $V_{\alpha}$ becomes a compact topological $T_{1}$-space.

If $\alpha=\left\{a_{1}, a_{2}, \ldots, a_{n}\right\}$ and if $\beta$ is a subset of $\alpha$, say if $\beta=\left\{a_{1}, a_{2}, \cdots, a_{m}\right\}, m<n$, then the projection $\pi_{\beta}^{\alpha}$ of $V_{\alpha}$ onto $V_{\beta}$ is given by the equations:

$$
X_{e_{1} e_{2} \cdots c_{m}}^{(\beta)}: X_{\delta_{1} \delta_{2} \cdots \delta_{m}}^{(\beta)}=X_{e_{1} \ell_{2} \cdots c_{m} \gamma_{m+1} \cdots \gamma_{n}}^{(\alpha)}: X_{\delta_{1} \delta_{2} \cdots \delta_{m} \gamma_{m+1} \cdots \gamma_{n}}^{(\alpha)},
$$


where each $\epsilon, \delta$ and $\gamma$ can take independently the values 1 or 2 . Thus $\pi_{\beta}^{\alpha}$ is a single-valued rational transformation of $V_{\alpha}$ onto $V_{\beta}$, and therefore $\pi_{\beta}^{\alpha}$ is closed and $\left(\pi_{\beta}^{\alpha}\right)^{-1}$ is open. It is clear that the closed sets in the infinite direct product $R^{*}$, as defined above, are the sets defined by (finite or infinite) systems of homogeneous equations, each equation involving the variables $X^{(\alpha)}$ relative to some finite subset $\alpha$ of $A$.

4. The space of homomorphic mappings of one abstract field into another. We now further specialize our application by assuming that the set $A$ is a field. The space $R^{*}$ is then the space of all single-valued transformations $x^{*}: a \rightarrow x_{a}=x_{a 1} / x_{a 2}$, of the field $A$ into the set consisting of the elements of the field $K$ and of the symbol $\infty$. We shall now express in an appropriate homogeneous form the conditions that a given mapping $x^{*}$ be a homomorphism. Let $\alpha$ be a subset of $A$ consisting of three elements, $\alpha=\left\{a_{1}, a_{2}, a_{3}\right\}$. On the corresponding variety $V_{\alpha}$ let $F_{a_{1}, a_{2}, a_{3}}$ be the algebraic subvariety obtained by imposing on the 6 parameters $x_{i 1}, x_{i 2}, i=a_{1}, a_{2}, a_{3}$, the following condition:

$$
x_{a_{1} 1} x_{a_{2} 2} x_{a_{3} 2}+x_{a_{1} 2} x_{a_{2} 1} x_{a_{3} 2}=x_{a_{1} 2} x_{a_{2} 2} x_{a_{3} 1} .
$$

Similarly we define another algebraic subvariety $G_{a_{1}, a_{2}, a_{3}}$ of $V_{\boldsymbol{\alpha}}$ by the equation

$$
x_{a_{1} 1} x_{a_{2} 1} x_{a_{3} 2}=x_{a_{1} 2} x_{a_{2} 2} x_{a_{3} 1} .
$$

Let $x_{a_{j 1} 1} / x_{a_{j} 2}=x_{j}, j=1,2,3$, where $x_{j}$ may be $\infty$. Suppose that equation (2) holds true. Then if $x_{1}$ and $x_{2}$ are both different from $\infty$ we find $x_{3}=x_{1}+x_{2}$. If $x_{1}=\infty$ and $x_{2} \neq \infty$, then $x_{a_{2}}=0, x_{a_{1}} \cdot x_{a_{2}} \neq 0$, whence (2) yields $x_{a_{2}}=0$, that is, $x_{3}=\infty$. Assume now that equation (3) holds. Again we find that $x_{3}=x_{1} x_{2}$, if both $x_{1}$ and $x_{2}$ are different from $\infty$. If $x_{1}=\infty$ and $x_{2} \neq 0$, then $x_{a_{1} 2}=0, x_{a_{1} 1} \cdot x_{a_{2} 1} \neq 0$, and (3) yields $x_{a_{3} 2}=0$, that is, $x_{3}=\infty$. Thus the equations (2) and (3) are the homogeneous counterparts of the equations $x_{3}=x_{1}+x_{2}$ and $x_{3}=x_{1} x_{2}$ respectively, and they include the conventions which are usually made for the symbol $\infty$. We can therefore assert that $x^{*}$ represents a homomorphic mapping of $A$ into $(K, \infty)$ if and only if the following conditions are satisfied: for any three elements $a_{1}, a_{2}, a_{3}$ of $A$ such that respectively $a_{3}=a_{1}+a_{2}$ or $a_{3}=a_{1} a_{2}$, the projection $\pi_{a}^{-1} x^{*}$ (where $\left.\alpha=\left\{a_{1}, a_{2}, a_{3}\right\}\right)$ must lie respectively on $F_{a_{1}, a_{2}, a_{3}}$ or on $G_{a_{1}, a_{2}, a_{3}}$. Therefore, if we denote by $\boldsymbol{M}$ the set of all homomorphic mappings of $A$ into $(K, \infty)$, we see that

$$
M=\bigcap_{\alpha} \pi_{\alpha}^{-1} F_{a_{1} a_{2} a_{8}} \bigcap_{\beta} \pi_{\beta}^{-1} G_{b_{1} b_{2} b_{8}},
$$


where the index $\alpha$ ranges over all sets $\alpha=\left\{a_{1}, a_{2}, a_{3}\right\}$ such that $a_{3}=a_{1}+a_{2}$, and the index $\beta$ ranges over the sets $\beta=\left\{b_{1}, b_{2}, b_{3}\right\}$ such that $b_{3}=b_{1} b_{2}$. We see thus that $M$ is an intersection of basic closed subsets of $R^{*}$. Hence $M$ is closed, and since $R^{*}$ is compact $M$ is also compact.

The case which is of special interest to us is that in which $K$ is a subfield of $A$. In this case we are interested in the relative homomorphisms of $A$ into $(K, \infty)$, that is, in the homomorphisms $x^{*}$ which leave each element of $K$ invariant. If $M^{*}$ is the set of all these relative homomorphisms, then it is clear that $M^{*}$ is the intersection of $M$ with the closed set $\bigcap_{a \in K} \pi_{a}^{-1} a$. Here, according to our notations, $\pi_{a}^{-1} a$ denotes that subset of $R^{*}$ which consists of the points $x^{*}$ whose $a$-component $x_{a}$ is $a$ itself $(a \in K)$. Hence also $M^{*}$ is a compact space.

It is convenient to describe in algebro-geometric terms the relative topology induced in $M^{*}$ by the topology of $M$. Let $x_{1}, x_{2}, \cdots, x_{n}$ be a finite set of elements of $A$. For each $x_{i}$ we introduce a pair of homogeneous parameters $x_{i 1}, x_{i 2}$ such that $x_{i 1} / x_{i 2}=x_{i}$. We consider the algebraic variety $Z$ over $K$ whose general point has as homogeneous coördinates the quantities $X_{(\epsilon)}$ defined by the parametric equations

$$
\rho X_{\epsilon_{1} \epsilon_{2} \cdots \epsilon_{n}}=x_{1 \epsilon_{1}} x_{2 \epsilon_{2}} \cdots x_{n \epsilon_{n}},
$$

where each $\epsilon_{j}$ can take the values 1 or 2 . If the quantities $x_{i}$ are algebraically independent, then the variety $Z$ coincides with the variety $V_{\alpha}$ defined by the equations (1), $\alpha$ being the subset $\left\{x_{1}, x_{2}, \cdots, x_{n}\right\}$ of $A$. But in general $Z$ is a subvariety of $V_{\alpha}$. If $x^{*} \in M^{*}$, then the mapping $x^{*}$ of $A$ into $(K, \infty)$ must preserve all the algebraic relations between $x_{1}, x_{2}, \cdots, x_{n}$ over $K$, since $x^{*}$ is a homomorphism. It follows that the point $\pi_{\alpha} x^{*}$ of $V_{\alpha}$ must lie on $Z$. Now we observe that the homomorphism $x^{*}$ defines a unique valuation of $A / K$ whose residue field is $K$ itself and whose center on $Z$ is the point $\pi_{\alpha} x^{*}$. Conversely, every valuation of $A / K$ whose residue field is $K$ defines a relative homomorphic mapping of $A / K$ onto $(K, \infty)$. We conclude that if $W$ is any algebraic subvariety of $Z$, then the set of all valuations of $A / K$ having $K$ as residue field and having center on $W$ is a closed subset of $M^{*}$. By taking different finite subsets $\left\{x_{1}, x_{2}, \cdots, x_{n}\right\}$ of $A$ and different subvarieties $W$ of $Z$ we obtain a family of closed subsets of $M^{*}$ which form a basis for the closed subsets of $M^{*}$.

REMARK. Suppose that $A$ is a field of algebraic functions in any number of variables, over a given ground field $k$. We identify the field $K$ with the algebraically closed field determined by $k$. The Riemann manifold $M$ of $A$ is the set of all zero-dimensional valuations $v$ 
of $A$. By the ground field extension $k \rightarrow K$ we can embed $A$ in a field $A^{\prime}=K A$. Every relative homomorphic mapping of $A^{\prime}$ onto $(K, \infty)$ determines uniquely a zero-dimensional valuation of $A^{\prime} / K$, and vice versa. Every zero-dimensional valuation of $A^{\prime} / K$ induces a unique zero-dimensional valuation of $A / K$, but a given zero-dimensional valuation of $A / K$ may be extendable in more than one way to a zerodimensional valuation of $A^{\prime} / K$. It follows that the Riemann manifold $M^{\prime}$ of $A^{\prime} / K$ coincides with the space $M^{*}$ of all relative homomorphic mappings of $A^{\prime}$ onto $(K, \infty)$ and is therefore a compact space. The Riemann manifold $M$ of $A / K$ is obtainable from $M^{\prime}$ by topological identification and therefore can also be converted into a compact topological space. That is precisely what we have proved in $\$ 2$.

The Johns Hopkins UnIVERSITy 